\title{
Estimativa do tamanho da trinca em corpo de prova com linha de solda: simulação $\mathrm{x}$ resultado experimental
}

\author{
Bom, Ricardo P.; Kalnin, Flávio A. ${ }^{\text {I }}$ \\ ${ }^{\mathrm{I}}$ Universidade do Estado de Santa Catarina - UDESC - Centro de Ciências Tecnológicas - CCT. Dept ${ }^{\mathrm{o}}$ Eng $^{\mathrm{a}}$ \\ Mecânica, Mestrado em Ciência e Engenharia de Materiais, Campus Universitário Avelino Marcante, B. \\ Bom Retiro, CEP 89.201-100, Joinville, SC, Brasil \\ e-mail: dem2rpb@joinville.udesc.br, flaviokalnin@expresso.com.br
}

\section{RESUMO}

Este trabalho tem por objetivo apresentar os resultados obtidos por simulação numérica realizada com o aplicativo desenvolvido neste laboratório, o qual permite prever o tamanho da trinca em corpo de prova com linha de solda. Os resultados da simulação serão comparados com os resultados experimentais obtidos a partir de corpos de prova moldados pelo processo de injeção em diferentes temperaturas e caracterizados a partir de ensaio de tração. $\mathrm{O}$ aumento do tamanho da trinca em produtos moldados pelo processo de injeção pode provocar a diminuição da tensão de ruptura efetiva na região de sua existência devido à diminuição da área de contato ou de interpenetração. Este problema pode ser de grande importância em peças estruturais, pois pode reduzir a tensão efetiva de trabalho.

A partir de equações que descrevem: o resfriamento de polímeros, a reologia de polímeros, a difusão e a interpenetração molecular e tomando-se como base o modelo de reptação descrito por De Gennes, determinou-se o perfil da difusão molecular na interface de junção dos fluxos e a interpenetração ao longo da espessura, bem como se determinou a interpenetração crítica utilizando-se o tamanho da trinca.

Por fim, com a diminuição da temperatura de injeção, o corpo de prova solidifica mais rapidamente, diminuindo a difusão e a interpenetração molecular na interface da linha de junção. Com o aumento da interpenetração molecular, aumenta-se a tensão máxima de ruptura do polímero termoplástico Polystyrol 158 K da Basf. Determinou-se uma dependência entre a interpenetração máxima no centro do corpo de provas e a interpenetração crítica correspondente à trinca obtida por ensaios de tração em função da variação da temperatura. Assim, foi desenvolvido um aplicativo que descreve com muita coerência a dimensão da trinca.

Palavras-chaves: Difusão molecular, tempo de resfriamento, linha de solda, interpenetração molecular.

\section{Crack size prediction in samples containing weld line: simulation $x$ experimental result}

\section{ABSTRACT}

This paper presents results from numeric simulation performed using a software specially developed to determine the crack length in samples with a weld line. The simulations results were compared with experimental results obtained by tensile testing samples molded by the injection process with several melting temperatures. The increase on the crack length in products made by the injection process may provoke a decrease on the effective rupture stress due to the decrease on the contact area or on the interpenetration. This problem might be a relevant one for structural parts since it might reduce the effective work stress.

Using appropriated equations to describe: polymer cooling and rheology, the molecular interdiffusion and interpenetration, and taking as a basis DeGennes's reptation model, it was possible to determine the inter-diffusion profile in the weld line interface and the interpenetration along the thickness. The critical interpenetration was determined on the basis of the crack length value.

Finally, reducing the injection temperature the sample solidifies faster, thus decreasing the molecular inter-diffusion and the interpenetration in the weld line interface. By increasing the molecular interpenetration the maximum rupture stress of the Basf thermoplastic polymer Polystyrol $158 \mathrm{~K}$ is also increased. The dependence between the maximum interpenetration in the middle of the sample and the critical interpenetration corresponding to the crack found by tensile testing the samples as a function of temperature variation was determined. Therefore, a software was developed able to well and coherently describe the crack dimension.

Keywords: molecular diffusion, cooling time, weld line, molecular interpenetration. 


\section{INTRODUÇÃO}

No processamento de polímeros, o termo linha de solda é usado para designar a interface criada quando dois fluxos de polímero se encontram dentro da cavidade do molde. Tal fenômeno é conseqüência direta da geometria do produto [1, 2] Segundo Doi \& Edwards [3], a reconstituição da interface criada pela linha de solda pode ser descrita pelo modelo do tubo aplicado a polímeros lineares. Modelo esse que teve seu início com os estudos de Rouse que, desprezando a interação intermolecular, contribuiu de maneira relevante para o entendimento da dinâmica molecular e seus parâmetros estatísticos. Outro pesquisador importante nessa área foi Zimm, que aplicou os conceitos de Rouse em soluções poliméricas, levando em consideração a interação solvente/polímero. De Gennes [4] é o nome mais conhecido, porque foi dele a conclusão de que a interação intermolecular cria um tubo imaginário que limita o caminho da molécula dentro da massa polimérica e, assim, estava sendo criado o modelo do tubo para a teoria da reptação.

Dentre os estudos sobre linha de solda, podem ser citados Koster [5] e Chang \& Faison [6], que analisaram as variáveis de maior relevância na reconstituição de uma interface de linha de solda, como velocidade de injeção, temperatura de injeção e do molde, tempo e pressão de recalque, pressão de injeção, contra-pressão e viscosidade.

Wool [7] e Pecorini [8] também utilizaram a difusão molecular em uma interface de linha de solda proposta por Doi \& Edwards para estudar sua reconstituição.

O’Brien-Soucy \& Carol \& Stacer [9] analisaram a redução da resistência em amostras com linha de solda em função da trinca produzida e Shaoyun \& Ait-Kadi \& Bousmina [10] também empreenderam esforços similares.

A linha de solda e as variações que esta produz nas propriedades do material têm prejudicado o desenvolvimento de muitos materiais com excelentes propriedades. Em função disto, tal fenômeno pode vir a ser um defeito, dependendo do material, do processo e da geometria, tornando-se o "calcanhar de Aquiles" para muitos materiais.

\section{MATERIAIS E MÉTODOS}

A solução analítica da Equação 1 pode ser encontrada em trabalhos recentes. Esta equação trata sobre o resfriamento de uma placa de material plástico a uma determinada temperatura no instante $\mathrm{t}=0$, a qual é aproximada por duas placas metálicas com temperatura constante e menor que a temperatura da placa plástica. No decorrer do tempo, a placa de material plástico vai resfriando e após um determinado tempo "t" a placa plástica passa a ter a mesma temperatura das placas metálicas. Dividindo a metade da espessura da placa plástica em várias camadas e considerando o centro da placa como $\mathrm{y}=0$, pode-se determinar analiticamente a partir da Equação 1 o perfil da temperatura em função da variação da espessura das camadas e do tempo de resfriamento [11]. Por semelhança, esta equação é utilizada com boa aproximação, para determinar o tempo de resfriamento, solidificação e desmoldagem de peças produzidas pelo processo de moldagem por injeção.

$$
\frac{\mathrm{T}_{\text {mol }}-\mathrm{T}_{(\mathrm{y}, \mathrm{t})}}{\mathrm{T}_{\text {mol }}-\mathrm{T}_{\text {inj }}}=2 \sum_{\mathrm{n}=\mathrm{o}}^{\infty} \frac{(-1)^{\mathrm{n}}}{\left(\mathrm{n}+\frac{1}{2}\right) \pi} \exp \left[-\left(\mathrm{n}+\frac{1}{2}\right)^{2} \pi^{2} \frac{\alpha \mathrm{t}}{\mathrm{e}^{2}}\right] \cos \left[\left(\mathrm{n}+\frac{1}{2}\right) \frac{\pi \mathrm{y}}{\mathrm{e}}\right]
$$

Onde: $\mathbf{T}_{\mathbf{m o l}}$ é a temperatura do molde, $\mathbf{T}_{\mathbf{i n j}}$ é a temperatura de injeção, $\mathbf{T}(\mathbf{y}, \mathbf{t})$ é uma temperatura entre a temperatura de injeção e a temperatura do molde para um determinado tempo de resfriamento " $t$ " e da camada analisada “y”. O tempo total de resfriamento "t" é o tempo necessário para que as paredes do produto possam atingir a temperatura do molde. O tempo de solidificação é o tempo no qual o material atinge a temperatura de transição vítrea "Tg" ou a temperatura de fusão “Tm”. Tempo de desmoldagem é o tempo necessário para abrir o molde e realizar a extração do produto sem ter distorção geométrica da peça. Neste caso, utiliza-se a temperatura de distorção do material, para o PS esta temperatura é de $90^{\circ} \mathrm{C}$. Em todos estes casos, o tempo foi determinado para o centro das paredes $(\mathrm{y}=0)$. Em um trabalho experimental de moldagem por injeção foi realizada a variação da pressão e do tempo de recalque para duas cavidades com forma de corpo-de-prova, conforme ASTM D 638. Cada cavidade tinha um canal de ataque próprio, um com 1,1 mm e outro com 2,2 mm. Determinou-se o aumento da massa dos corpos de prova e dos canais de distribuição. No instante em que os canais de ataque solidificaram, as massas dos corpos de prova não se alteram. O tempo de recalque em que isto ocorreu é coerente com o tempo de solidificação do canal de ataque determinado por diversas equações inclusive pela Equação $1[12,13]$. Kalnin [2] avaliou o resultado analítico da Equação 1, 
no que diz respeito à variação da temperatura em função do tempo, com o resultado obtido com um simulador de fluxo, as diferenças dos valores foram inferiores a 1,8\%.

Existe um parâmetro intrínseco do material que é a difusividade térmica $(\alpha)$, que pode ser determinada a partir da Equação 2, apesar de ser considerada constante na Equação 1. A difusividade também depende da variação da temperatura. Os demais parâmetros são: condutividade térmica (K) para o PS utilizado, $\mathrm{k}=0,140 \mathrm{~W} / \mathrm{m}^{\mathrm{O}} \mathrm{C}$, massa específica $(\rho)$ foi considerada constante para o material plastificado, $\rho=0,955 \mathrm{~g} / \mathrm{cm}^{3}$ e o calor específico do material $\left(C_{p}\right)$ como sendo $C_{p}=1.970 \mathrm{~J} / \mathrm{kg}^{\mathrm{O}} \mathrm{C}$.

$$
\alpha=\frac{k}{\rho C p}
$$

Foi desenvolvido, neste laboratório, um aplicativo denominado “CrackDim” em linguagem de programação Visual Basic, que resolve as Equações 1 e 2. O formato da janela de entrada de dados, assim como suas respectivas unidades, estão indicados na Figura 1. Os dados de entrada correspondem a parâmetros de processo, assim como propriedades intrínsecas do polímero termoplástico de estrutura amorfa, designado comercialmente por Polystyrol $158 \mathrm{~K}$, produzido pela Basf.

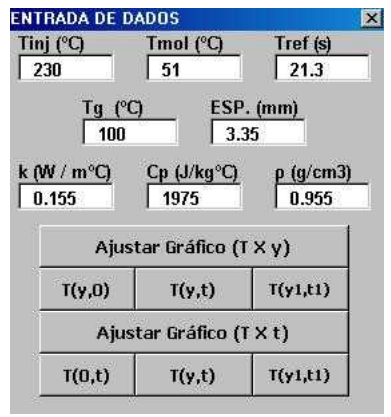

Figura 1: Janela de entrada de dados para solução da Equação 1.

Nas respectivas janelas da Figura 1, foram configuradas com os seguintes parâmetros de entrada: temperatura de injeção (Tinj), temperatura de transição vítrea (Tg), temperatura do molde (Tmol), condutividade térmica $(\mathrm{k})$, calor específico (Cp), massa específica $(\rho)$, espessura do corpo-de-prova (ESP.) e Tref (s) que é o tempo de resfriamento o qual se deseja observar na figura. "CrackDim".

As Figuras 2 e 3 apresentam as soluções dadas pelas Equações 1 e 2 realizadas pelo aplicativo

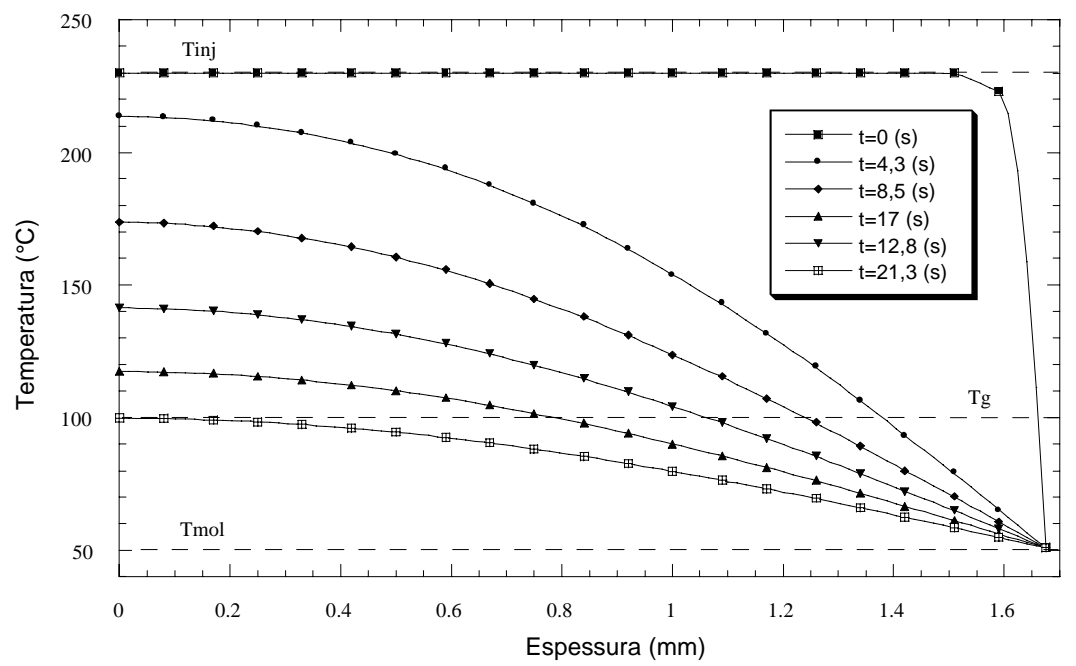

Figura 2: Variação da temperatura em função da espessura do corpo-de-prova para diversos tempos de resfriamento 
Na Figura 2, estão representadas as variações da temperatura de resfriamento em função da espessura para seis intervalos de tempo de resfriamento e, na Figura 3, está representada a variação da temperatura de resfriamento em função do tempo de resfriamento. Sendo indicadas nesta figura, seis curvas com intervalos de tempo definidos indicam a variação da temperatura para cada posição do centro $(\mathrm{y}=0)$ até a face externa do corpo-de-prova $(\mathrm{y}=1,675 \mathrm{~mm})$. Como pode ser visto nesta figura, para o instante $\mathrm{t}=0$, a superfície que está encostada no molde metálico resfria rapidamente, convergindo à temperatura do molde. À medida que o tempo vai passando, as linhas isócronas descrevem o comportamento da temperatura para cada camada. Raciocínio semelhante está representado na Figura 3, onde está indicada a variação da temperatura em função do tempo de resfriamento. No caso da interface molde/face externa ( $y=1,675 \mathrm{~mm})$, o corpo de prova atingiu rapidamente a temperatura do molde e a curva se sobrepõe a reta indicado por $\mathrm{T}_{\mathrm{mol}}$.

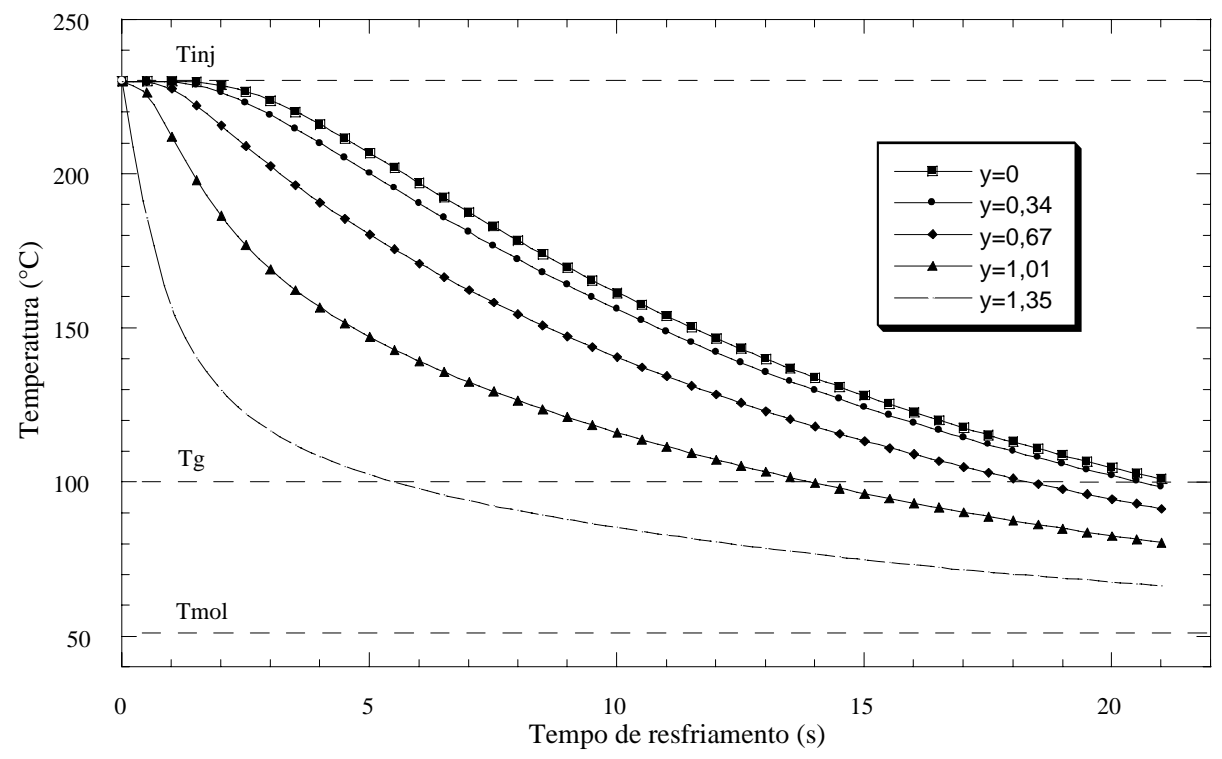

Figura 3: Variação da temperatura em função do tempo de resfriamento para varias camadas da espessura

Na Figura 3, fica clara a identificação dos tempos de solidificação, pois a interseção entre a reta da Tg com as demais curvas permite determinar qual o tempo de solidificação de cada camada. Deve-se levar em conta que a superfície da peça atinge instantaneamente a temperatura do molde, então para y=1,675 mm e $\mathrm{t}=0 \mathrm{~s}, \mathrm{~T}=\mathrm{Tmol}$. A camada central terá um tempo maior de resfriamento do que as demais.

Conhecendo a temperatura, o tempo de solidificação e o número de camadas, pode-se calcular a difusão molecular na interface de linha de junção dos fluxos a partir da Equação 3. Segundo Wool [7] é preciso haver primeiro: rearranjo das superfícies, aproximação, molhabilidade, a difusão e a randomização das macromoléculas, para se obter uma boa reconstituição da interface.

$$
D_{G}=\left(\frac{\rho R T}{270}\right)\left(\frac{M_{C}}{M}\right)^{2}\left(\frac{R_{g}^{2}}{M}\right)\left(\frac{M}{M_{C}}\right)^{3,4}\left(\frac{1}{\eta_{0}(M)}\right)
$$

Os termos da Equação 3 são os seguintes: a massa específica ( $\rho)$, a constante universal dos gases $(\mathrm{R})$, a temperatura (T), a massa molar (M), a massa molar crítica (Mc), o raio de giração (Rg) e a viscosidade

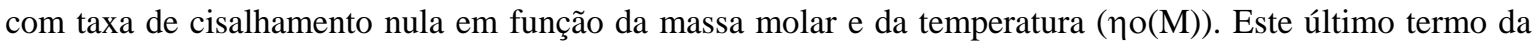
Equação 3 pode ser descrito pela expressão de WLF [7], conforme Equação 4, podendo os parâmetros desta equação serem obtidos na literatura e nas bibliotecas dos simuladores de fluxo.

$$
\eta_{0}(T)=D_{1} \exp \left[\frac{-A_{1}(T-\dot{T})}{A_{2}+(T-\dot{T})}\right]
$$

As constantes da equação acima são: $\mathrm{D}_{1}=2,25 \cdot 10^{12} ; \mathrm{A}_{1}=28,3 ; \mathrm{A}_{2}=51,6$, fonte C-mold [14]. 
Inserindo a Equação 4 na Equação 3, tem-se como resultado a equação geral do coeficiente de difusão dado pela Equação 5. As temperaturas utilizadas nesta equação deverão ser as mesmas da solução da Equação 1, isto porque, fica condicionado o respectivo tempo de resfriamento à temperatura e ao coeficiente de difusão.

$$
D_{G}=\left(\frac{\rho R T}{270}\right)\left(\frac{M_{C}}{M}\right)^{2}\left(\frac{R_{g}^{2}}{M}\right)\left(\frac{M}{M_{C}}\right)^{3,4}\left(\frac{1}{2,25.10^{12} \exp \left[\frac{-28,3(T-373,15)}{51,6+(T-373,15)}\right]}\right)
$$

Para se calcular a interpenetração $(\chi)$ para cada camada, conforme Equação 6, é necessário calcular a área abaixo da curva de Dg x t. O resultado é multiplicado por dois e extraído a raiz quadrada. A Figura 4 apresenta a variação do coeficiente de difusão em função do tempo de difusão para y=0 e para uma temperatura de injeção de $230{ }^{\circ} \mathrm{C}$. Deve-se notar que o tempo de difusão é o mesmo que o tempo de solidificação. Este procedimento deve ser realizado para todas as camadas e em todas as temperaturas de injeção. O resultado da interpenetração em função da espessura está indicado na Figura 5.

$$
\chi^{2}=2 D_{G} \cdot t
$$

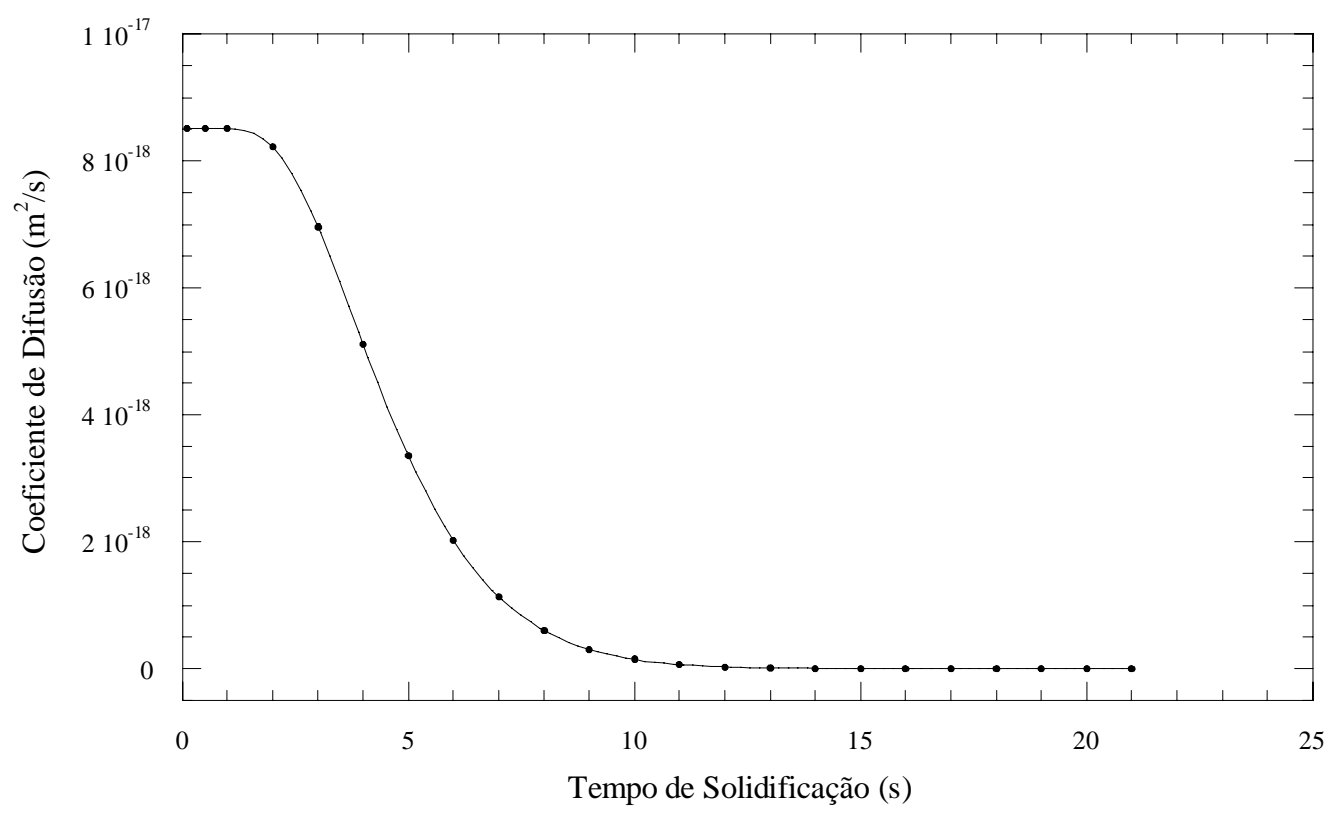

Figura 4: Variação do coeficiente de difusão em função do tempo, $\mathrm{T}=230^{\circ} \mathrm{C}, \mathrm{y}=0$.

Pode-se observar que, no centro do corpo-de-prova, a interpenetração é máxima e designada $(\chi \infty)$. Na região próxima à parede do molde, onde a temperatura da superfície do corpo de prova resfria, rapidamente, a interpenetração é nula. É interessante determinar um $\Delta$ t adequado para evitar a formação de um ombro na curva próximo a $0,2 \mathrm{~mm}$ da parede. 


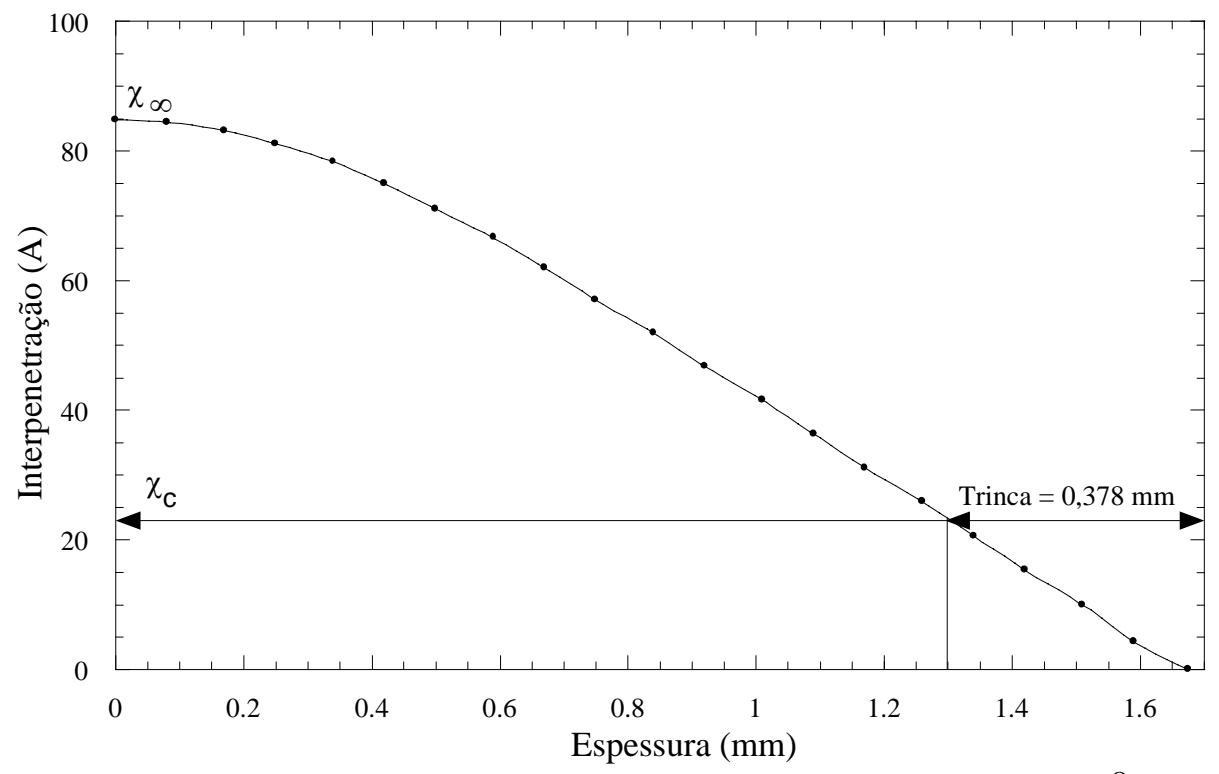

Figura 5: Variação da interpenetração em função da espessura, para $\mathrm{T}=230^{\circ} \mathrm{C}$

A partir de corpos de provas ASTM D 638 moldados pelo processo de injeção e caracterizados por ensaio mecânico de tração, conforme trabalho desenvolvido por Kalnin [2], cujos resultados estão descritos na Tabela 1, foi possível, com ajuda da Equação 7, determinar as dimensões das trincas efetivas e os seus respectivos desvios padrão.

$$
4 a^{2}-32,7 a+43,55\left(1-\frac{\sigma_{w}}{43,6}\right)=0
$$

Medindo a trinca da parede externa do corpo de prova sentido centro, a interseção com a curva de interpenetração, como indicado na Figura 5, permite determinar o parâmetro de interpenetração crítico $(\chi c)$, sendo que a interpenetração máxima ocorre no centro da placa. Os valores, assim determinados para a interpenetração máxima e crítica para todas as temperaturas de ensaio, estão indicados na Tabela 1.

Tabela 1: Valores da temperatura de injeção, $\sigma_{\omega}, \sigma_{0}$, trinca e respectivos desvios padrão, parâmetro de interpenetração crítico $\left(\chi_{C}\right)$ e parâmetro de interpenetração máximo $\left(\chi_{\infty}\right)$

\begin{tabular}{ccccccc}
\hline $\begin{array}{c}\text { Temperatura de injeção } \\
\left({ }^{\circ} \mathrm{C}\right)\end{array}$ & $\begin{array}{c}\sigma_{\omega} \\
(\mathrm{MPa})\end{array}$ & $\begin{array}{c}\text { Desvio } \\
\text { Padrão } \\
(\mathrm{MPa})\end{array}$ & $\begin{array}{c}\text { Trinca } \\
(\mathrm{mm})\end{array}$ & $\begin{array}{c}\text { Desvio } \\
\text { Padrão } \\
(\mathrm{mm})\end{array}$ & $\begin{array}{c}\chi_{c} \\
(\AA)\end{array}$ & $\begin{array}{c}\chi_{\infty} \\
(\AA)\end{array}$ \\
\hline 180,0 & 22,56 & 2,37 & 0,703 & 0,086 & 8,17 & 16,49 \\
204,0 & 26,74 & 0,74 & 0,552 & 0,026 & 16,24 & 41,02 \\
215,0 & 28,82 & 1,32 & 0,479 & 0,045 & 19,87 & 57,27 \\
230,0 & 31,77 & 1,06 & 0,378 & 0,036 & 23,59 & 84,84 \\
245,0 & 34,57 & 1,25 & 0,285 & 0,041 & 24,94 & 118,75 \\
260,0 & 37,04 & 1,74 & 0,205 & 0,056 & 24,19 & 158,91 \\
280,0 & 38,65 & 1,33 & 0,154 & 0,042 & 23,16 & 221,81 \\
Sem linha de solda & $\sigma_{0}=43,60$ & 1,04 & 0,000 & & & \\
\hline
\end{tabular}

A Figura 6 representa a relação entre o parâmetro de interação crítico e máximo em função da variação da temperatura de injeção. 


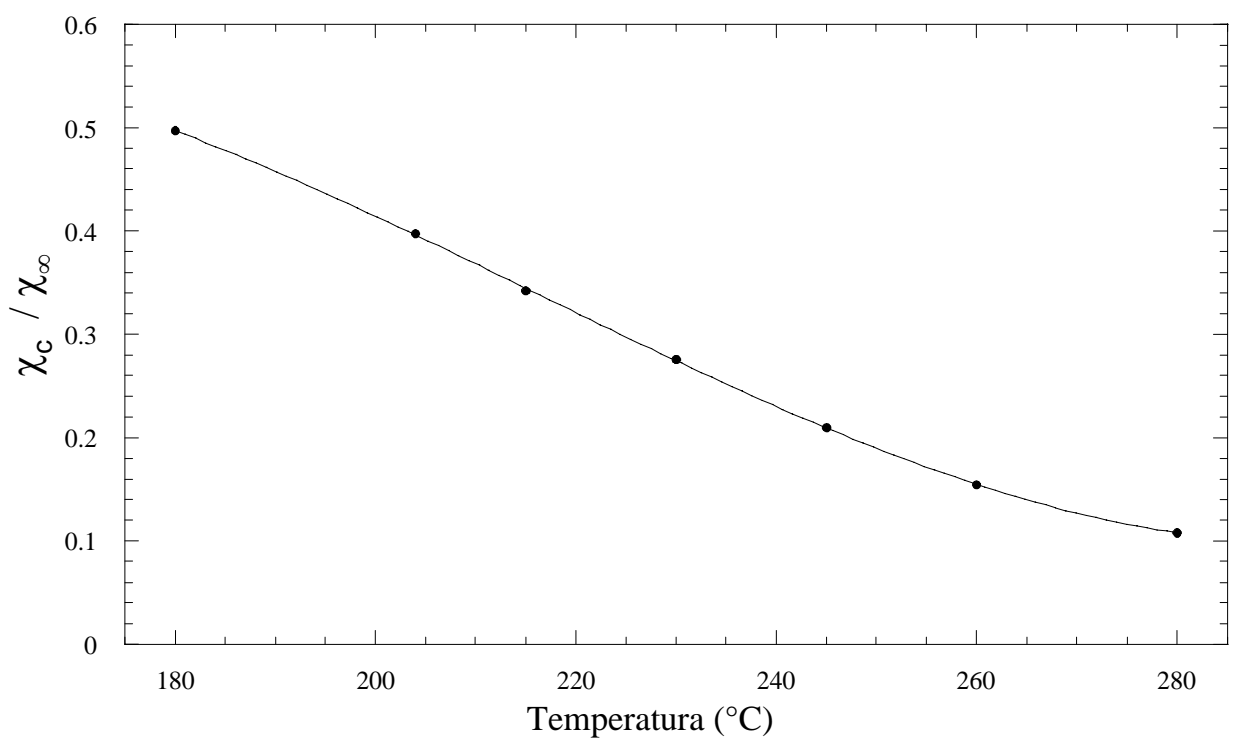

Figura 6: Relação entre o parâmetro de interação crítico e máximo em função da temperatura de injeção

Como pode ser observado, à medida que a temperatura aumenta a relação entre $\chi \mathrm{c} / \chi \infty$ decresce. Isto significa que o parâmetro de interpenetração crítico aproxima-se do valor máximo, ou seja, melhora a interpenetração da interface. É interessante este comportamento, visto que pode ser descrito por um polinômio do terceiro grau e em função da temperatura de injeção, conforme indicado pela Equação 8. Desta forma, pode-se obter o valor da interpenetração crítica para temperaturas de processamento intermediárias.

$$
\frac{\chi_{c}}{\chi_{\infty}}=-1,861+0,039513 T-0,00020225 T^{2}+3,0803 \cdot 10^{-7} T^{3}
$$

As Equações 5, 6 e 8 estão inseridas no aplicativo “CrackDim” e a continuação da Figura 1 é a Figura 7. Os valores utilizados estão indicados na figura: a massa molar $(\mathrm{M})$, a massa do monômero $\left(\mathrm{m}_{0}\right)$, a massa crítica $\left(\mathrm{M}_{\mathrm{c}}\right)$ e o raio de giração $\left(\mathrm{R}_{\mathrm{G}}\right)$ este último calculado pela Equação 9.

$$
\mathrm{R}_{\mathrm{G}}^{2}=\frac{\mathrm{N} \cdot \mathrm{b}^{2}}{6}
$$

Onde "N" é o grau de polimerização e igual a $N=M / m_{0}$, "b" é o comprimento do mero, b=2,45 $\AA$. Obtém-se como resultado, ao pressionar a tecla INTERPENETRAÇÃO ( $\AA$ ) , a curva indicada pela Figura 8. Para uma dada temperatura de injeção, o aplicativo pode calcular o tamanho da trinca.

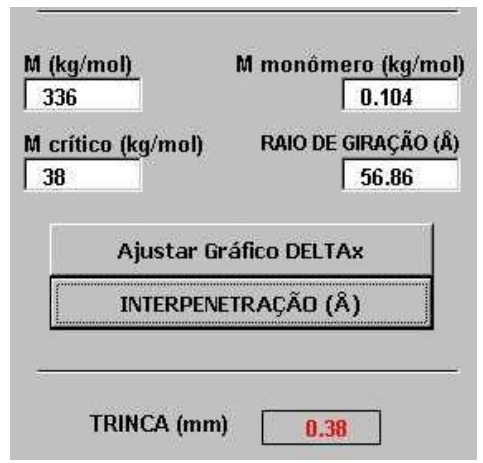

Figura 7: Janela de entrada de dados para cálculo do tamanho da trinca 


\section{RESULTADOS E DISCUSSÃO}

O resultado final da simulação realizada com o aplicativo “CrackDim” está representado na Figura 8, onde os pontos experimentais estão indicados com seus respectivos desvios padrão. Os demais pontos e a curva spline fazem parte do resultado apresentado pelo aplicativo. Como pôde ser visto, o programa descreve e prevê coerentemente o tamanho da trinca para temperaturas de processamento compreendidas entre 180 e $280^{\circ} \mathrm{C}$. Variando-se a temperatura do molde para mais até $70^{\circ} \mathrm{C}$ ou para menos até $20^{\circ} \mathrm{C}$, surgirão duas curvas: uma abaixo e outra acima da curva estabelecida para $51^{\mathrm{O}} \mathrm{C}$. Entretanto, estas curvas estão muito próximas entre si e dentro do desvio padrão dos valores experimentais, com variação máxima de $\pm 0,012 \mathrm{~mm}$. Seria interessante realizar ensaios com corpos de prova com espessuras diferentes para estabelecer a relação entre os principais parâmetros como: temperatura de injeção e do molde com o tamanho da trinca, esta última, obtida a partir de ensaios de tração. Segundo Kalnin, o percentual de redução da tensão de ruptura foi de $11,35 \%$ para a temperatura de $280^{\circ} \mathrm{C}$ e de $48,26 \%$ para a temperatura de $180^{\circ} \mathrm{C}$ [2].

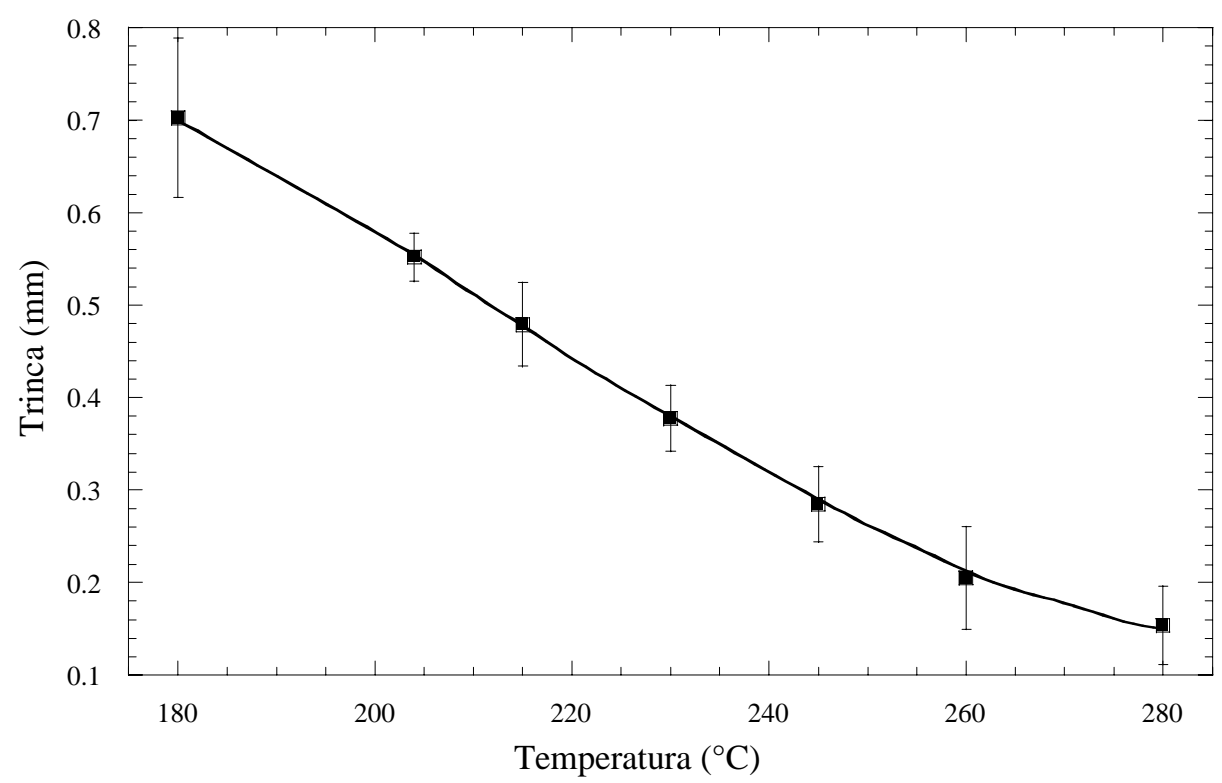

Figura 8: Comparação entre resultados experimentais e simulados, predição do tamanho da trinca, em função da variação da temperatura.

\section{CONCLUSÕES}

Nesta primeira etapa do desenvolvimento, deste trabalho, foi possível reproduzir e, até mesmo, estimar a dimensão da trinca para toda e qualquer temperatura de injeção compreendida entre 180 e $280^{\circ} \mathrm{C}$. Foram utilizadas equações que tratam do resfriamento de placas, que descrevem o coeficiente de difusão e a interpenetração molecular. Os resultados obtidos com o aplicativo "CrackDim” foram coerentes com os resultados experimentais. O aplicativo também permitiu calcular o tamanho da trinca para diferentes temperaturas do molde, ao aumentar a temperatura reduziu-se a dimensão da trinca, diminuindo-se a temperatura aumenta a trinca. Os desvios padrão calculados para as trincas experimentais foram muito importantes e as estimativas do tamanho das trincas em função da variação da temperatura do molde são muito próximas. Foi possível, graças à relação entre a interpenetração máxima e crítica em função da variação da temperatura, estabelecer uma relação entre a teoria da reptação e a trinca em corpos de prova que possibilitou descrever o resultado experimental. A equação foi obtida a partir de resultados experimentais de ensaios de tração aplicados apropriadamente nos gráficos de interpenetração. Uma das principais contribuições deste trabalho foi a sua aplicação em problemas práticos. Os resultados que foram apresentados permitirão aos profissionais que atuam na área de projetos de produtos ou que atuam na área de cálculo estrutural ou simulação de fluxo estimar e quantificar a dimensão da trinca bem como a tensão de ruptura do material na região da linha de solda a partir de parâmetros de processo e do produto.

\section{REFERÊNCIAS BIBLIOGRÁFICAS}

[1] PINTO, M.A.G., BOM, R.P., "Aspectos estéticos e estruturais de termoplásticos injetados: linhas de junção e de solda fria”, Plástico Industrial, ano 3, n. 37, pp. 146-153, setembro de 2001. 
[2] KALNIN, F.A., Análise da perda de resistência a tração em amostras com linha de solda, M.Sc, Ciência e Engenharia de Materiais, Universidade do Estado de Santa Catarina, Centro de Ciências Tecnológicas, pp.160, Joinville, 2006.

[3] DOI, M., EDWARDS, S.F., The theory of polymer dynamics, 1 ed., Nova York, Oxford University Press, 1986.

[4] DE GENNES, P.G., Scaling concepts in polymers physics, 1 ed., Nova York, Cornell University Press, 1979.

[5] KOSTER, R.P., "Importance of injection molding parameters for mechanical performance of cold flow weld line”, The Journal of injection molding technology, v. 3, n 3, pp. 154-158, 1999.

[6] CHANG, T., FAISON, E., "Optimization of weld line in injection molding via an experimental design approach”, The Journal of injection molding technology, v. 3, n 2, pp. 61-66, 1999.

[7] WOOL, R.P., O’ CONNOR, K.M., “A Theory of Crack Healing in Polymers”, Journal Applied Physics, v. 52(10), pp. 5963, Outubro, 1981.

[8] PECORINI, T.J., "A fracture mechanics approach to weld line fracture in a amorphous cellulose acetate propionate”, Polymer Engineering and Science, v. 37, n. 2, pp. 308-314, 1997.

[9] O'BRIEN-SOUCY, A.K., BARRY, C.M.F., STACER R.G., The development of weld line strength in injection molded PMMA, University of Massachusetts, 1999

[10] SHAOYUN, G., AIT-KADI, A., BOUSMINA, M.A., "A modified model predictions and experimental results of weld line strength in injection molded PS/PMMA blends”, Elsevier, 2004.

[11] AGASSANT, J.F., AVENAS, P., SERGENT, J.P., VICENT, M., "La Mise em Forme des Matières Plastiques”, 3 ed., Paris, Ed. Tec. \& Doc. Lavoisier, pp.137-147, 1996.

[12] DA CRUZ, A.C., Determinação do Tempo de Solidificação de Peças Termoplásticas Moldadas pelo Processo de Injeção, M.Sc, Ciência e Engenharia de Materiais no Centro de Ciências Tecnológicas, Universidade do Estado de Santa Catarina, UDESC, pp. 21 e 69, Julho 2005.

[13] BOM, R.P., Efeito da Pressão e do Tempo de Recalque no Empacotamento de Peças Injetadas, Monografia apresentada para a obtenção do Título de Especialista em Ciência e Engenharia de Materiais no Centro de Ciências Tecnológicas, Universidade do Estado de Santa Catarina, UDESC, pp. 22-27, Junho 2002.

[14] C-mold. C-mold Design Guide. Product Number D-701, Version 969.0498. Copyright@ 1994-1998 Advanced CAE Technology, Inc. 